\title{
Virtual Reality Nature Exposure and Test Anxiety
}

\author{
Alison $O^{\prime}$ Meara *(D), Marica Cassarino ${ }^{\circledR}$, Aaron Bolger ${ }^{\circledR}$ and Annalisa Setti * \\ School of Applied Psychology, University College Cork, T23 TK30 Cork, Ireland; mcassarino@ucc.ie (M.C.); \\ aaron.bolger@ucc.ie (A.B.) \\ * Correspondence: 117323401@umail.ucc.ie (A.O.); a.setti@ucc.ie (A.S.)
}

Received: 24 August 2020; Accepted: 20 October 2020; Published: 22 October 2020

\begin{abstract}
The number of students affected by exam anxiety continues to rise. Therefore, it is becoming progressively relevant to explore innovative remediation strategies that will help mitigate the debilitating effects of exam anxiety. The study aimed to investigate whether green environment exposure, delivered by virtual reality (VR) technology, would serve as an effective intervention to mitigate participants' test anxiety and therefore improve the experience of the exam, measured by positive and negative affect, and increase test scores in a pseudo exam. Twenty high and twenty low exam anxiety students completed a pseudo exam before and after being exposed to either a simulated green environment or urban environment. Only those who had high anxiety and were exposed to the nature VR intervention had significant reductions in negative affect $(F(1,31)=5.86$, $p=0.02, \eta_{p}{ }^{2}=0.15$ ), supporting the idea that exposure to nature, even if simulated, may benefit students' feelings about their academic performance. The findings are discussed in light of future developments in nature and educational research.
\end{abstract}

Keywords: green environments; exam anxiety; virtual reality; academic performance

\section{Introduction}

An extensive body of literature asserts that exposure to green environments can help combat attentional fatigue, cognitive overload and alleviate stress [1] due to their restorative properties [2]. The term 'green environments' denotes any area of nature consisting of vegetated regions such as parks, forests and open landscapes [1]. Two prominent theories, namely attention restoration theory (ART) [3,4] and stress recovery theory (SRT) [5] are proposed to explain the restorative mechanisms behind green environments.

Modern life requires constant attentional engagement, resulting in eventual mental fatigue [3,4]. ART asserts that green environments elicit an alternative form of effortless attention within individuals, described as 'soft fascination,' i.e., the effortless exploration of an interesting but not attentionally demanding environment [3]. Green environments also evoke a sense of 'being away' from attentional demands, feelings of extent (i.e., existing in a big enough world where it is possible to perceive 'scope,' 'coherence') [6]. Such restorative factors of green environments work together with 'soft fascination' to facilitate the replenishment of an individual's depleted levels of attention.

This restoration of attention is reflected in performance improvements in memory and attention-related tasks and suggests that our cognitive performance can be beneficially impacted by nature exposure [4].

SRT asserts that green environments elicit restorative cognitive benefits due to their relationship with human evolution [5]. SRT argues that because humans possess this intrinsic tie to the natural world, exposure to green environments can positively stimulate our parasympathetic nervous system in a way that elicits a reduction in stress and arousal levels [5]. In agreement with SRT, taking a walk through a forest or other natural environments has been shown to cause significant reductions in cortisol levels amongst young adults [7]. 
Seminal research carried out by Berto [8] found that university students who viewed photos of nature before completing a sustained attention task scored better compared to those who viewed photos of an urban landscape or geometric patterns [8], therefore, not only 'real' nature, but also virtual nature possess restorative properties. Such restoration obtained from exposure to green environments can be achieved through a variety of mediums including being physically present in nature, window views and viewing photographs, and other forms of virtual reality $[9,10]$. Despite the variety of durations of exposure to nature, from spending several days experiencing nature to observing photos for just a few minutes, each produce positive results in relation to mental stress and attentional fatigue [9-11].

Importantly, contemporary developments in immersive virtual reality (IVR), have facilitated the creation and customisation of virtual green environments to increase availability of nature benefits to individuals who may not have immediate access to it [12]. Works by Ulrich et al. [5] and de Kort et al. [13] have demonstrated that the restorative qualities of green environments can be evoked by 'virtual nature' through the use of videos of real green environments. Immersive virtual environments (IVEs) are synthesised sensory information that produces a continuous and surrounding flow of stimuli [12]. IVEs create an illusory perception of being encompassed by an authentic environment [12]. Research conducted by Calogiuri et al. in 2018 [12] investigated whether commercial IVE technology could be used in a laboratory condition to stimulate a green exercise experience. The participants scored the immersive virtual environments with adequate levels of "sense of reality" and "being there," indicating that IVE has the potential to serve as a useful technology for green environment research and practice [12]. Moreover, accumulating research has validated the use of IVE technology as a therapeutic tool for managing stress and promoting relaxation [14-17]. Valtchanov, Barton and Ellard [15] found that individuals exhibited an increase in positive affect and a reduction in stress post-green-environment exposure via IVR compared to controls.

As nature, real or virtual, can alleviate stress and improve cognitive performance, one context in which its benefits could be applied is test anxiety in relation to academic performance [18]. Nowadays, testing has become an intrinsic part of society, particularly within academic life [19]. Throughout secondary school and college, students' academic status is largely determined by their performance in examinations [20]. Unsurprisingly, a sizeable number of students are affected by test anxiety. Test anxiety can be described as the dread, fear and worry of failure that occurs during or before testing scenarios [19]. It is induced by the collection of tension, physiological over-arousal and somatic symptoms which often leads to poor concentration, detrimental cognitive appraisals and uncomfortable physiological responses [20]. There is a widespread consensus in the empirical literature that high test anxiety is a rigorous predictor of lower student academic performance [21]. Therefore, students that possess the capability to succeed in exams, often perform poorly due to the incapacitating effects of exam anxiety [22]. There is also evidence of a negative association between students' high-test anxiety and academic success [23-25]. Moreover, several worldwide studies have revealed that the number of students suffering from anxiety disorders, including test anxiety, continues to rise [26]. Therefore, it is becoming progressively relevant to explore innovative methods that will serve to mitigate the debilitating effects of exam anxiety.

Research evidence highlights a significant association between exposure to green environments and reductions in anxiety and its precursors [1]. For example, Parnabas and Mahamood found that athletes who were the most frequent users of green imagery visualisation experienced the least amount of performance anxiety and vice versa [27]. The precise mechanism by which green environment exposure helps to alleviate anxiety remains under investigation [28]. One mechanism proposes that green environment exposure elicits a decrease in ruminative thoughts [29]. This mechanism has been supported by a recent neuroimaging study which found that a ninety-minute walk in nature elicited a significant decrease in both subgenual prefrontal cortex ( $\mathrm{sgPFC}$ ) activity and self-reported measures of rumination. The sgPFC is a brain region that is found to be active during ruminative thoughts, therefore, a decline in activity in this area is associated with a decline in rumination [29]. 
In the context of exam anxiety, distraction theories posit that excessive pressure during exam scenarios can elicit ruminative thoughts in anxious individuals [30]. Test-taking relies heavily on working memory and requires the majority or all of one's attentional resources [31]. Test performance can become impaired when such resources are strained by task-irrelevant thoughts (e.g., ruminative thoughts). This explains why anxious individuals often 'choke' under the pressure of the exam scenario [31]. It is therefore possible that, in line with SRT and ART, exposure to a green environment may serve to alleviate the task overload that is experienced by individuals with test anxiety and thereby help to improve their performance.

The current literature on green environments and academic performance rests mainly on looking at the beneficial effects of available green areas in a school context [18]. Leung et al. [32] found a consistent positive link between greenness within school surroundings and academic performance (based on academic performance \% or composite performance index). Canteen and classroom views that have a higher quantity of shrubs and schools with higher tree counts have been linked with better test scores and graduation rates [33,34]. Interestingly, 'featureless landscapes,' consisting of vast areas of land such as athletic fields or campus lawns, had a negative impact on student performance in line with ART [34]. These studies claim that when students reach a state of mental exhaustion, they are likely to become irritable and have difficultly performing and concentrating on basic tasks. Green environments can provide a restorative antidote for such attentional fatigue [18,32-34]. However, there is a lack of research on whether nature exposure, real or virtual, can contribute to alleviating test anxiety, specifically in an academic context.

The present study aims to investigate whether one stint of nature exposure through IVR can improve the experience of the test and test performance in individuals with test anxiety. We chose IVR as many individuals 'choke' under the pressure of being in an exam hall [30], where nature is not available. Therefore, our study represents a first step in determining whether technology can support students' experience of exams by virtual exposure to natural environments immediately before a test.

Two hypotheses were examined. Firstly, that high test anxiety participants' self-reported negative affect would be lower following exposure to a green environment via virtual reality and that this change would not be observed by participants who are exposed to an urban environment via virtual reality. Second, we aimed to test whether positive affect was increased by virtual exploration of a natural scene. We chose to assess positive and negative affect as they are related to exam anxiety and they have been shown to impact the effect on test anxiety on performance [35] and can be measured as a state before and after the test. Negative affect in particular, is associated with test anxiety and academic performance [35]. It was anticipated, based on the arguments of the aforementioned ART and SRT theories, that the restorative properties of a green environment, as opposed to an urban environment, would facilitate the reduction in negative affect that is associated with exam anxiety [3-5] as well as increase positive affect, when conveyed by IVR. Thirdly, it was hypothesised that participants' test performance i.e., test scores would improve following exposure to a green environment via virtual reality and that this change would not be observed by participants who are exposed to an urban environment via virtual reality. It was envisioned that because participant's levels of negative affect would be reduced following the intervention that this in turn would improve the participant's overall ability to concentrate and focus on the exam.

\section{Materials and Methods}

\subsection{Participants}

Participants were recruited via convenience sampling. The sample was restricted to students of the Higher Education Institution of the research team. As shown in Supplementary File 2, participants included in the final sample for the high anxiety group had mean age of 20.5 (SD =1.36), similarly participants in the low anxiety group had mean age of $20.3(\mathrm{SD}=0.92)$. The two groups were also similar in terms of average number of years in higher education. Firstly, two separate emails 
(see Supplementary File 1) were sent out via two university emailing lists and social media platforms, one aimed at recruiting students who felt they have high test anxiety and one aimed at recruiting students who felt they do not have high test anxiety. The email s also contained a link to fill out a brief demographics and nature connection index (NCI) [36] survey and also highlighted the study's aim and detailed the inclusion criteria for participation. Inclusion criteria were that students were aged 18 years old or older, felt that they experience a high level of test anxiety but they are not seeking any clinical treatment for it, i.e., not a clinical population (for the email target to high anxiety students). The second email was sent out aimed to recruit the control population. The content of this email was the same as the aforementioned email with the exception that it sought to recruit individuals who have healthy or low levels of exam anxiety (i.e., individuals who do not feel that they experience particularly high levels of test anxiety). This first step of recruitment had the scope of estimate whether there was an interest in the study from students who recognised themselves as having low or high anxiety, before proceeding with the administration of the test anxiety questionnaire.

In the second step of recruitment, an email was sent out to students who had responded to the first email and completed the demographics survey. This email instructed the participants to fill out a test anxiety questionnaire [37]. Based on the results of the test anxiety questionnaire, quartiles were calculated and only those who scored within the upper quartile (score 35/50 and above) or lower quartile (score 24/50 and below) of the test anxiety questionnaire were contacted to arrange a time to complete the experimental session.

As a final attempt to recruit additional high anxiety participants, a second email was sent out to those who initially completed the demographics survey but did not fill out the test anxiety survey.

Based on an a priori G-Power analysis, this study aimed to recruit a total of eighty participants (forty per group). However, this target was not reached due to the time constraints of this research, including the impossibility of protracted recruitment due to the Covid-19 lockdown. A total of forty university students within the age range of 18-24 were successfully recruited, twenty who met the inclusion criteria for the high anxiety group (males $=4$, females $=16$ ) and twenty who met the inclusion criteria for the low anxiety group (males $=6$, females $=14$ ). Although we acknowledge that this is a limitation of this study, we refer to the effect sizes (medium to large) and observed power to support the validity of our results in this pilot study, which will require replication.

Ethical approval was sought for and granted by the UCC Ethics Committee of the School of Applied Psychology on the 29/11/2019 (ID number: 1211201923). Any possible stress that the participants may have experienced when completing the verbal reasoning test was no greater, or at the most only as great as, the degree of stress they would be subjected to under a real-life exam scenario. Nonetheless, extra care was taken to ensure that participants were reminded that it was only a pseudo test and that it would not impact their academic grades or determine their abilities in any way. Given that this was a pilot study, we felt a more cautious approach to inducing anxiety than simulating an actual exam would be suitable as first step in determining the efficacy of our intervention. The project was conducted in accordance with the Declaration of Helsinki and adhered to the code of professional ethics as according to the Psychological Society of Ireland. This research also adhered to standard G.D.P.R regulations regarding data acquisition and storage.

\subsection{Design}

The present research employed a quantitative mixed between and within-participants design ( 2 (high vs. low anxiety) $\times 2$ (urban vs. nature virtual reality $(\mathrm{VR})) \times 2$ (pre vs. post VR intervention) There was an experimental (high anxiety) and a control (low anxiety) group. Participants were assigned to one of the two groups based on their scorings on the test anxiety questionnaire [37]. Within each group, participants were randomly assigned to either an urban or nature virtual reality (VR) intervention condition. The dependent variables were the positive and negative affect schedule, PANAS [38] and the test performance scores obtained pre- and post-urban or rural VR intervention. 


\subsection{Materials}

All measures included in this study, together with a rationale for choosing these measures, are presented in Supplementary File 2.

\subsubsection{Test Anxiety Questionnaire}

A test anxiety questionnaire by Nist and Diehl [37] was used to evaluate participants' baseline levels of test anxiety. This six-item questionnaire was chosen as it is regarded as a brief yet validated and standardised measure of test anxiety [39].

\subsubsection{Positive and Negative Affect Schedule (PANAS)}

The PANAS is a self-report psychometric scale [38] that consists of two 10-item scales. PANAS has been empirically deemed to be a trustworthy and robust instrument for evaluating individual levels of positive affect (PA) and negative affect (NA) by numerous clinical and non-clinical studies [40,41]. Therefore, the PANAS was deemed to be an appropriate measure for assessing participants' momentary feelings towards taking an exam pre- and post-intervention.

\subsubsection{Non-Verbal Reasoning Test}

The non-verbal reasoning test was used to measure participants' test scores pre- and post-intervention [42]. Results from six pilot sessions indicated that this test posed a sufficient, comprehensible challenge to third-level students. None of the individuals that took part in the pilot session took part in the experimental session. The content of this test is not directed towards any specific subject or academic skills (e.g., maths or English), and therefore a fair test for all participants with different academic capabilities. While there was no particular time constraint placed on how long they had to complete this test, each participant was instructed to complete the test as promptly as they could. The test was answered by means of multiple-choice questions on an answer sheet provided.

\subsubsection{Demographics Questionnaire}

The demographics questionnaire served as a means of collecting information relating to participant sex, age, years of third-level education, living environment and previous virtual reality experience. 'Living environment' denoted whether a participant lived in a mostly urban or rural environment, 'VR prior' denoted whether a participant has used VR technology prior to taking part in this experiment and 'years of education' denoted the number of years each participant had attended university. These covariates were measured to determine if place of residence, familiarity with VR, length of university attendance or motion sickness would have any impact on the significance of anxiety levels (i.e., positive and negative affect scores) or test performance (i.e., test scores) following the VR intervention.

\subsubsection{Nature Connection Index (NCI)}

This self-reported questionnaire by Richardson et al. [36] was used to capture individual differences in affinity towards nature which was regarded as a potential mediating factor concerning the efficacy of the nature intervention technique. The NCI is considered to be a brief, yet valid and reliable tool for measuring nature connectedness [36].

\subsubsection{Motion Sickness Assessment Questionnaire (MSAQ)}

The MSAQ [43] acted as a means of identifying any the group of symptoms including for, e.g., nausea and dizziness associated with the discrepancy between sensory inputs potentially experienced by participants during the virtual reality intervention. 


\subsubsection{Virtual Reality Footage}

The nature intervention condition consisted of a 360-degree experience of being immersed in a lush green forest, populated with pine trees and a small stream of flowing water. The footage for this intervention was taken in Coombaune Woods, Kenmare, Co. Kerry, Ireland https://www.youtube.com/

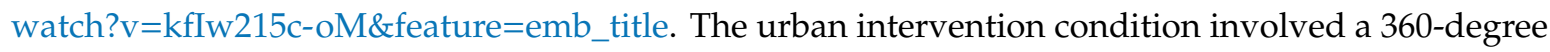
experience of being immersed in an alleyway of an urban town, this scene mainly consisted of the back of buildings and a parked car. The duration of the videos was set to 4 minutes based on pilot work described below. The footage for this intervention was taken in Killorglin, Co. Kerry, Ireland. The accompanying sounds generated by both environments were also recorded. The footage for both conditions was recorded on a GoPro Fusion 360-degree camera. There is considerable variation in the literature of green environment exposure and virtual reality (VR) regarding their time frame [15-17]. Therefore, the VR green environment was piloted by 6 third-level students, who were recruited by convenience sampling among class-mates/acquaintances of the first author; they were of similar age of the experimental sample. Three of these individuals piloted the VR for five minutes, while the other three piloted the VR for ten minutes. Overall, individuals generally indicated that they were ready to stop watching the video after three to five minutes therefore, it was decided that for this research the VR intervention would be of a four-minute duration. None of these individuals involved in the pilot sessions took part in the experimental sessions. Furthermore, a pilot study conducted by Tarrant, Viczko and Cope [44] which is similar to the present study, used 360-degree videography to simulate a nature-based mindfulness virtual reality experience that was found to elicit significant reductions in state anxiety [44]. The VR session of this experiment lasted 5 minutes and 41 seconds; therefore, this further substantiated the decision to proceed with the shorter time frame of 4 minutes as indicated by the pilot study.

\subsubsection{Virtual Reality Equipment}

The virtual reality interventions were operated on an Alienware 86 computer via GoPro fusion software and delivered to the participant via immersive virtual environment (IVE) technology. IVE was chosen as it has been deemed to be a cost-efficient and effective mode of technology for green environment research [1]. The IVE technology consisted of an 'HTC Vive' headset and accompanying headphones.

\subsection{Procedure}

Prior to the experimental session, participants filled out the aforementioned demographics and NCI questionnaire and the test anxiety questionnaire. All participants provided written consent to take part before completing the surveys (information and consent sheet are presented in Supplementary File 3).

The experimental session took place in a university research lab in the presence of a researcher. For the experimental session, participants were assigned a randomly generated three-digit participant number. Participants were first briefed regarding what the experiment would entail. Then, participants began by filling out the PANAS. Participants were instructed to answer this questionnaire in response to how they were feeling in the present moment, with a particular reference to their feelings towards having to complete an exam afterward. Following this, participants completed the first session of the non-verbal reasoning test. Upon completion of this test, participants underwent the virtual reality (VR) intervention. Participants remained seated while taking part in the VR intervention. Depending on which condition they had been assigned to, participants had four minutes to observe either an urban or nature environment. Participants were seated in a swivel chair, and so were able to move their head and the orientation of the chair in whatever direction they pleased to explore the 360-degree nature of the environment. Participants were also asked to wear headphones so that they could listen to the accompanying noise of the environment. Participants then completed the PANAS questionnaire for a second time with the same principles in mind as when they completed the first 
PANAS. Participants were then given the second session of the non-verbal reasoning test to complete. Finally, participants were asked to fill out the MSAQ questionnaire in relation to their VR intervention experience. The researcher was present in the room throughout the experimental session.

Upon completion of the experiment, participants were fully debriefed about the nature of the experiment and to what group they had been assigned. Any participant queries were answered.

\subsection{Data Analysis}

Statistical analyses were conducted using version 26 of IBM SPSS. Assumptions for mixed ANOVA were assessed using the Shapiro-Wilk test and also by inspecting the box plots, Q-Q plots and 5\% trimmed means of each independent variable. The Levene's test was used to assess homogeneity of variance for positive affect (PA) scores, negative affect (NA) scores and test scores. The means and standard deviations for each group of participants (high anxiety nature, high anxiety urban, low anxiety nature, low anxiety urban) were determined for PA scores, NA scores and test scores preand post-virtual reality intervention. In order to match the design of the study, each of our dependent variables (PA, NA and test scores) was analysed by performing a 2 (high vs. low anxiety) $\times 2$ (nature vs. urban) $\times 2$ (pre vs. post) mixed ANOVAs with nature connectedness, living environment, VR prior, years of education and motion sickness as covariates. The ANOVAs were conducted without and with the covariates to check changes in the effect of the predictor when controlling for potential confounding factors. Post-hoc pairwise comparisons were conducted using independent samples $t$-test and paired samples t-test. A Bonferroni correction was applied $(p=0.012)$ to correct the alpha level to reduce the possibility of a Type 1 error [45].

\section{Results}

\subsection{Preliminary Analyses}

Considering assumptions for the Mixed ANOVA, the assumption of normality of standardised residuals, tested using the Shapiro-Wilk test, was met for positive affect (TTime 1, $p=0.42$; TTime 2, $p=0.47$ ) and for test scores (Time 1, $p=0.15$; Time 2, $p=0.15$ ), but not for negative affect (Time 1, $p=0.003$; Time 2, $p=0.015$ ); in the case of negative affect, inspection of box plots, Q-Q plots and the mean vs. $5 \%$ trimmed mean indicated no potential outliers, thus, the measure was treated as continuous. The assumption of homogeneity of variance tested through the Levene's test was also met for all measures (positive affect: Time $1 p=0.33$, Time $2 p=0.97$; negative affect: Time $1 p=0.57$, Time $2 p=0.17$; t test scores: Time $1 p=0.71$, Time $2 p=0.51$ ). A Maulchy's test of sphericity was not conducted due to the study having two sessions only. However, to adjust for potential breaches of sphericity, the Wilk's lambda adjusted F-ratio was used to consider the statistical significance of within-subjects interaction and main effects.

\subsection{Inferential Statistics}

The high anxiety and low anxiety groups were similar in terms of age, years of education, NCI and prior experience with VR. The majority of participants in the high anxiety group reported to live in an urban environment $(n=14)$ whereas many of the low anxiety participants lived in a rural place $(n=12)$. The demographics scores are presented in Supplementary File 4 . Table 1 presents the results for each group and condition. 
Table 1. Means and Standard Deviations for Positive Affect, Negative Affect and Test Scores.

\begin{tabular}{ccccccccccc}
\hline & & \multicolumn{4}{c}{ HA } & \multicolumn{3}{c}{ LA } \\
\cline { 3 - 11 } & & \multicolumn{2}{c}{ T1 } & & T2 & & T1 & & T2 \\
\cline { 3 - 11 } & & M & SD & M & SD & M & SD & M & SD \\
\hline Positive & Nature & 27.80 & 8.59 & 30.80 & 8.94 & 32.00 & 5.57 & 32.60 & 6.68 \\
Affect & Urban & 26.80 & 5.69 & 26.90 & 6.98 & 31.60 & 5.56 & 28.00 & 9.63 \\
\hline Negative & Nature & 27.20 & 9.35 & 15.50 & 4.90 & 13.60 & 4.01 & 11.60 & 2.83 \\
Affect & Urban & 21.50 & 8.55 & 16.90 & 5.06 & 13.90 & 3.51 & 11.50 & 2.32 \\
\hline \multirow{2}{*}{ Test Scores } & Nature & 5.20 & 2.25 & 5.40 & 2.17 & 5.60 & 1.83 & 5.40 & 2.06 \\
& Urban & 5.50 & 1.58 & 5.20 & 1.98 & 5.80 & 2.14 & 6.20 & 1.68 \\
\hline
\end{tabular}

Note: 'HA' denotes the high anxiety group while 'LA' denotes the low anxiety group. 'T1' denotes scores pre-intervention and 'T2' denotes scores post-intervention. 'Nature' and 'urban' denote the virtual reality intervention conditions.

\subsubsection{Positive Affect Scores}

Results from the mixed ANOVA conducted without covariates revealed that for the within-subject effects, there was no significant main effect of time (T1, T2) on PA scores, $F(1,36)=0.01, p=0.98$, $\eta_{p}{ }^{2}<0.01$. There was no significant interaction effect between time and anxiety, $F(1,36)=2.16, p=0.15$, $\eta_{p}{ }^{2}=0.06$, or between time and VR condition, $F(1,36)=2.93, p=0.09, \eta_{p}{ }^{2}=0.07$, on PA scores. Moreover, there was no significant interaction effect between time and anxiety and condition on PA scores, $F(1,36)=0.37, p=0.55, \eta_{p}{ }^{2}=0.01$. There were no significant between-subject effects.

When the ANOVA was run with covariates, there was still no significant main effect of time (T1T2) on PA scores, $F(1,31)=2.87, p=0.1, \eta_{p}{ }^{2}=0.08$. There was also no significant interaction effect between time and anxiety, $F(1,31)=2.62, p=0.11, \eta_{p}{ }^{2}=0.07$, or between time and VR condition, $F(1,31)=3.19$, $p=0.08, \eta_{p}{ }^{2}=0.09$, on PA scores. Moreover, there was no significant interaction effect between time and anxiety and condition on PA scores, $F(1,31)=0.04, p=0.83, \eta_{p}{ }^{2}<0.01$. As for the covariates, there was a significant interaction effect between time and motion sickness scores with a large effect size [46], $F(1,31)=4.49, p=0.04, \eta_{p}{ }^{2}=0.12$. There were no significant between-subject effects.

\subsubsection{Negative Affect Scores}

For the mixed ANOVA without covariates, there was a significant interaction effect between time and anxiety with a medium effect size [46], $F(1,36)=8.74, p=0.005, \eta_{p}^{2}=0.19$, observed power $=0.82$. There was also a significant main effect of time with a large effect size [46], $F(1,36)=26.47, p<0.001$, $\eta_{p}{ }^{2}=0.42$, observed power $=0.99$. There was no significant interaction effect between time and condition, $F(1,36)=2.77, p=0.10, \eta_{p}^{2}=0.07$ or between time, anxiety and condition, $F(1,36)=3.47$, $\mathrm{p}=0.07, \eta_{p}{ }^{2}=0.08$. For the between subject effects there was only a significant main effect for anxiety with a large effect size [46], $F(1,36)=27.16, p<0.001, \eta_{p}{ }^{2}=0.43$, observed power $=0.99$.

When the ANOVA was conducted with covariates, there was a significant three-way interaction between time ${ }^{*}$ anxiety* ${ }^{*}$ condition, with a moderate effect size, which explains $15 \%$ of the variance in negative affect [46], $F(1,31)=5.86, p=0.02, \eta_{p}{ }^{2}=0.15$, observed power $=0.66$ (see Figure 1 ). There was also a significant interaction between time and anxiety time with a large effect size [46], $F(1,31)=13.42$, $p=0.001, \eta_{p}{ }^{2}=0.30$, observed power $=0.95$. There was no significant effect for time, $F(1,31)=0.72$, $p=0.40, \eta_{p}^{2}=0.02$, or between time and condition, $F(1,31)=2.08, p=0.15, \eta_{p}{ }^{2}=0.06$. As for the covariates, there was only a significant interaction between the covariate living environment and time with a large effect size [46], $F(1,31)=5.96, p=0.02, \eta_{p}^{2}=0.16$, observed power $=0.67$. For the between-subject effects, there was a significant main effect of anxiety with a large effect size [46], $F(1,31)=21.08, p=<0.001, \eta_{p}{ }^{2}=0.40$, observed power $=0.99$. There was also a significant effect of the covariate VR prior with a large effect size [46], $F(1,31)=5.39, p=0.27, \eta_{p}^{2}=0.15$, observed 
power $=0.63$. No significant effects were observed for condition, $F(1,31)=0.01, p=0.95, \eta_{p}^{2}<0.001$, or between anxiety and condition, $F(1,31)=1.05, p=0.31, \eta_{p}^{2}=0.03$.

Negative affect in high anxiety Nature vs. Urban groups pre-post exposure

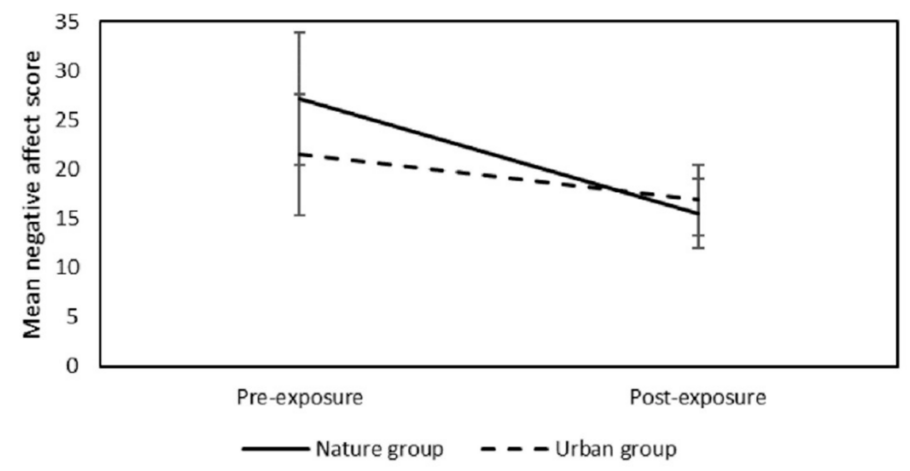

Figure 1. Line plot for mean scores of negative affect pre (Time 1) and post (Time 2) intervention in high anxiety participants, stratified by nature and urban exposure group. Error bars represent a $95 \%$ confidence interval.

As can be seen in Figure 1 below, there was considerable variation between the baseline (pre-exposure) scores between the high anxiety nature and urban group.

To accommodate for this, a factorial ANOVA was performed on adjusted baseline scores for negative affect [(NA Time 1-NA Time 2)/NA 1]. This yielded an interaction effect between anxiety*condition with a p-value of 0.051 .

Post-hoc comparisons using paired t-tests indicated that only the high anxiety nature group showed a significant reduction in negative affect scores from TTime $1(M=27.20, S D=9.35)$ to TTime 2 $(M=15.50, S D=4.90), t(9)=4.56, p=0.001$ (two-tailed). The Cohen's d value was 1.44 , which indicated a large effect size [46].

As can be seen in Figure 2a below, there was considerable variation in the differences in negative affect scores between TTime 1 and TTime 2 for each participant within the high anxiety nature group. However, mean difference scores within the high anxiety nature group were consistently higher than in the high anxiety urban group (Figure $2 b$ ) and showed greater overall declines across participants compared to this group in line with the significant effect on NA scores.
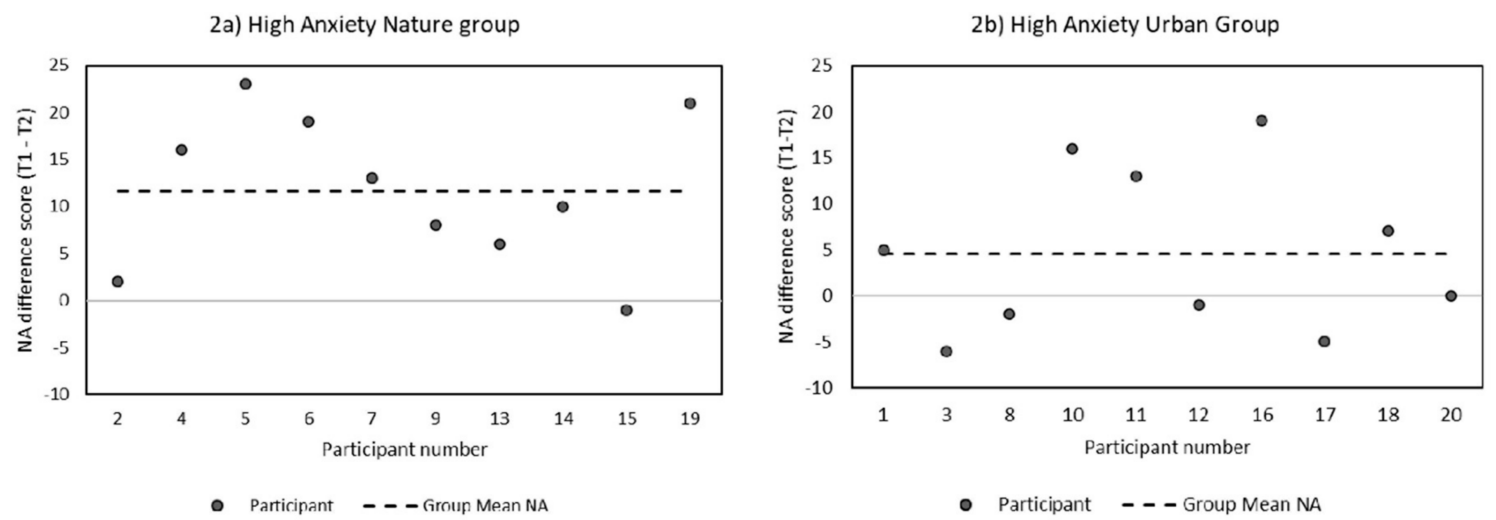

Figure 2. Scatter plots of pre-post differences in negative affect (NA) scores per participant in (a) the high anxiety nature group and (b) the high anxiety urban group. The dashed line represents the overall mean NA difference score for the group. 
In order to investigate the differences between groups, an independent-samples T-test was carried out. It was revealed that NA scores for the low and high anxiety groups were significantly different from one another at Time 1 with a large effect size, $d=1.51, t(38)=4.78, p<0.001$, (two-tailed) and Time 2 with a large effect size [46], $d=1.19, t(38)=3.77, p=0.001$, (two-tailed).

To assess where the differences were amongst the interaction effect between the covariate living (rural vs urban) and time, two paired-samples T-tests were carried out with a Bonferroni adjustment of $p=0.025$. It was revealed that both living in an urban or rural setting yielded significant impacts on negative affect scores post-VR intervention, $t(21)=2.60, p=0.01$, (two-tailed), with a moderate effect size, $d=0.55$ (see [46] for classification of effect size), and $t(17)=3.81, p=0.001$, (two-tailed), with a large effect size [46] $d=0.89$, respectively. In addition, participants who lived in a rural environment showed a greater decline in means from TTime 1 to TTime 2 (NA1 rural $M=19.22, S D=10.19$; NA 1 urban $M=18.90, S D=7.62$ : NA2 rural $M=12.27, S D=3.10 ; \mathrm{NA} 2$ urban $M=15.18, S D=5.10$ ).

\subsubsection{Test Scores}

For the mixed ANOVA without covariates, within-subject effects revealed that there was no significant main effect of time (T1, T2) on test scores, $F(1,36)=0.01, p=0.93, \eta_{p}^{2}<0.001$. There was also no significant interaction effect between time and anxiety, $F(1,36)=0.06, p=0.79, \eta_{p}{ }^{2}=0.01$, or between time and VR condition, $F(1,36)=0.007, p=0.83, \eta_{p}{ }^{2}=0.001$, on test scores. Moreover, there was no significant interaction effect between time and anxiety and condition on test scores, $F(1,32)=0.37, p=0.55, \eta_{p}^{2}=0.01$. There were no significant between-subject effects on test scores.

When the ANOVA was conducted with covariates, within-subject effects still revealed no significant main effect of time (T1, T2) on test scores, $F(1,31)=1.36, p=0.25, \eta_{p}^{2}=0.04$. There was no significant interaction effect between time and anxiety, $F(1,31)=0.001, p=0.97, \eta_{p}{ }^{2}<0.001$, or between time and VR condition, $F(1,31)=0.24, p=0.62, \eta_{p}{ }^{2}=0.01$, on test scores. Moreover, there was no significant interaction effect between time and anxiety and condition on test scores, $F(1,31)=1.28, p=0.26$, $\eta_{p}{ }^{2}=0.04$. There were no significant interactions for any of the covariates (years of education, living, VR prior, nature connection index and motion sickness). There were also no significant between-subject effects on test scores.

\section{Discussion}

\subsection{Positive and Negative Affect}

As hypothesised, for the NA scores it was found that only those who had high anxiety and were exposed to the nature VR intervention had significant reductions in negative affect scores. This indicates that, when controlling for relevant covariates, IVR nature exposure can reduce negative affect, which is associated with high exam anxiety [35], in high anxiety students. Due to the significant difference in baseline (TTime 1) scores between the high anxiety groups, it may be argued that the extent to which conclusions can be drawn from the significant difference in NA scores post- intervention within the high anxiety nature group is limited. In an attempt to correct for the baseline variance, a factorial ANOVA was performed on adjusted baseline scores [(NA TTime 1 - NA TTime 2) / NA 1] which held significance at the 0.05 level. Furthermore, the effect size for the interaction between time, anxiety and condition was moderate, as was the significant effect for the within subjects effect for the high anxiety nature group. Considering that significant moderate effects were detected, this further supports the validity of these findings. It remains a question for future studies whether variations in the intervention, for e.g., a longer duration, may allow for a restorative effect to emerge also in the low anxiety group.

Our study shows that individuals with high exam anxiety can lower their negative affect, which importantly, is associated with exam anxiety and its detrimental effects [35]. Moreover, four minutes of IVR nature exposure was sufficient to induce a reduction in negative affect. This significant finding extends the literature on IVE technology as an effective therapeutic tool for managing stress $[14,15,17]$. 
We believe that the reduction in negative affect highlights the potential for IVR technology to be used as a tool to alleviate negative affect associated with the experience of exams in a student population.

Anecdotally, several high anxiety participants that were exposed to the nature intervention reported feeling refreshed, as if their mind had been completely cleared of all the worrying thoughts they had about taking the test. These were unsolicited comments which the experimenter noted and they were not collected, nor analysed as a qualitative component of the study, which would be useful to introduce in the future. This type of commentary was not reported by any other group. Therefore, it is plausible that both the reduction in negative affect scores and the positive verbal response of the high anxiety participants exposed to the nature condition may have been achieved by a reduction in worrying, ruminative thought, in line with distraction theories and the beneficial effect of nature on ruminative thoughts [29]. Moreover, this proposed mechanism complements the widely accepted attention restoration theory (ART) [4]. The nature intervention may have elicited soft fascination which enabled the replenishment of their cognitive resources, resulting in them feeling refreshed and clear-minded. The cause of this, again, linked with the reduction in NA scores, may also be accounted for by the stress reduction theory (SRT) [5]. Further research could include a systematic record of subjective experience of the different types of IVR exposure.

The lack of a significant increase in PA scores following the nature-based virtual reality intervention contradicts the literature that has found increases in positive affect and mood [15,17] following VR exposure to natural environments. It is possible that a longer exposure time would have increased the chance to obtain an effect on positive affect, however the short intervention time was linked to avoiding major feelings of motion sickness. Further research is needed on the association between IVR green exposure and positive affect. Motion or 'cyber sickness' is a commonly reported issue amongst virtual reality research [12]. Calogiuri et al. notes that a better understanding of the factors that underlie cyber sickness is needed in order to figure out how to prevent it. Therefore, future studies may benefit from addressing this issue [12].

Alternatively, it is possible that positive affect does not capture the relationship between test anxiety and affect [35]. Several prominent existing interventions for the treatment of anxiety view reductions in negative affect as the central goal of treatment [47]. Moreover, some researchers have shown that despite a reduction in anxiety levels, individuals failed to show any concomitant changes in positive affect [48]. Nonetheless, mounting evidence argues that positive affect might be a valuable target in the treatment of anxiety [47] and that several variants of anxiety have been linked with low positive affect [49]. Therefore, further research could seek to clarify this association.

\subsection{Test Scores}

The second hypothesis stated that participants' test performance i.e., test scores would improve following exposure to a green environment via IVR and that this change would not be observed by participants who are exposed to an urban environment via IVR. Despite a promising positive association within the literature between green environment long-term exposure and academic performance [32-34], no significant effect between test scores and VR intervention was found. Several factors could have contributed to this non-significant finding.

Firstly, it is possible that the pseudo exam (non-verbal reasoning test) was too easy. Although this test was piloted by six third-level students and reported to be sufficiently difficult, the average test scores across each of the four subgroups pre-intervention $(5.2,5.5,5.6,5.8)$ and post-intervention $(5.4,5.2,5.4,6.2)$ indicate that participant's performance was at a good and consistent level throughout the experiment. Secondly, it is possible that the context of the pseudo test was too different from that of the test content that participants would be accustomed to under real-life testing scenarios (e.g., essays, calculations, etc.). Therefore, it may have been the case that the observed change in negative affect was simply not related to participants' capability to perform well on the non-verbal reasoning test. Given the nature of the experiment and its motivation to comply with the University code of professional ethics, this issue was hard to control for. Furthermore, participants were not placed under a short time 
constraint when completing the test. This was done to ensure that those with high exam anxiety did not get too overwhelmed by the pseudo exam experience and also to ensure that participants did not respond sporadically in an attempt to comply with time constraints. However, this in turn may have resulted in the test lacking endurance and the pressure associated with a real-life testing situation.

\subsection{Limitations}

The study however has some limitations; the baseline differences between groups existed even if both the high anxiety and low anxiety participants were randomly assigned to either VR condition, it is thought that future research with a larger sample size may not encounter this issue.

As mentioned, it is possible that the non-verbal reasoning test did not adequately simulate a 'real' exam scenario, or the test itself was not sufficiently sensitive to short term interventions. Future studies could benefit from replicating this study in a mock exam context. In this instance, participants would complete an authentic exam that pertains to their particular college course and carry out this exam in an authentic environment (e.g., lecture room, exam hall). While this exam scenario would appear 'real' to participants, they would be ensured that it was a pseudo exam that was not going to contribute to their grades. In addition, the authors selected the green and urban sceneries; in future studies it would be useful to pilot test different kinds of urban and natural environments for their restorative potential with a separate group of participants in order to obtain a more nuanced classification of restorative and non-restorative videos.

This study obtained data solely via self-reported measures. Moreover, there was no measure of how participants were feeling during the intervention. It is thought that the use of a device that provides biofeedback, such as those measuring electrodermal activity/galvanic skin response, may be of benefit to future research.

\subsection{Implications of the Study}

This study revealed a promising link between exposure to a green environment via virtual reality and the reduction in feelings of negative affect for students with high exam anxiety before a testing situation. This study demonstrated how an immersive virtual reality technology that integrates visual and auditory stimuli can be utilised to simulate an authentic and restorative experience of being immersed in a green environment. This indicates a potentially useful tool to apply to exam situations in an academic context. It is thought that further research seeking to replicate this study would help to validate this finding and extend it by utilising different tests and other more portable VR technology. Its applicability to other populations such as secondary school children could also be tested. It was observed that participants' commentary on how they felt about their experience of the virtual reality intervention proved to be very informative. Future studies could benefit from adding a brief interview section to the existing procedure as a means of enriching the statistical analyses.

If this intervention were proved to be continually effective under more realistic testing circumstances such as a mock exam, this intervention may have the potential to serve as a first step towards a cost-effective and accessible therapeutic tool that could help students to cope with surges in exam anxiety before a testing scenario.

Supplementary Materials: The following are available online at http://www.mdpi.com/2414-4088/4/4/75/s1, Supplementary File 1: high anxiety recruitment email, Supplementary File 2: Materials and Measures, Supplementary File 3: information sheet and consent form, Supplementary File 4: participant demographics.

Author Contributions: Study design and conceptualization A.O., A.S.; software A.B.; data analysis A.O., M.C.; draft of the manuscript A.O.; revisions A.S., M.C. All authors have read and agreed to the published version of the manuscript.

Funding: This research received no external funding, the study was conducted as a final year project for the BA in Applied Psychology by A.O., supervised by A.S.

Acknowledgments: The authors would also like to acknowledge Kathy Bradley, coordinator at the Skills Centre at University College Cork, for her assistance in the recruitment of participants for this project. 
Conflicts of Interest: The authors declare no conflict of interest.

\section{References}

1. Berto, R. The role of nature in coping with psycho-physiological stress: A literature review on restorativeness. Behav. Sci. 2014, 4, 394-409. [CrossRef] [PubMed]

2. Hartig, T.; Staats, H. Restorative environments. Encycl. Appl. Psychol. 2014, 3, 273-279.

3. Kaplan, S. Meditation, restoration, and the management of mental fatigue. Environ. Behav. 2001, 33, 480-506. [CrossRef]

4. Kaplan, R.; Kaplan, S. The Experience of Nature: A Psychological Perspective; CUP Archive: Cambridge, UK, 1989.

5. Ulrich, R.S.; Simons, R.F.; Losito, B.D.; Fiorito, E.; Miles, M.A.; Zelson, M. Stress recovery during exposure to natural and urban environments. J. Environ. Psychol. 1991, 11, 201-230. [CrossRef]

6. Hartig, T.; Mang, M.; Evans, G.W. Restorative effects of natural environment experiences. Environ. Behav. 1991, 23, 3-26. [CrossRef]

7. Tyrväinen, L.; Ojala, A.; Korpela, K.; Lanki, T.; Tsunetsugu, Y.; Kagawa, T. The influence of urban green environments on stress relief measures: A field experiment. J. Environ. Psychol. 2014, 38, 1-9. [CrossRef]

8. Berto, R. Exposure to restorative environments helps restore attentional capacity. J. Environ. Psychol. 2005, 25, 249-259. [CrossRef]

9. Bratman, G.N.; Daily, G.C.; Levy, B.J.; Gross, J.J. The benefits of nature experience: Improved affect and cognition. Landsc. Urban Plan. 2015, 138, 41-50. [CrossRef]

10. Hedblom, M.; Gunnarsson, B.; Iravani, B.; Knez, I.; Schaefer, M.; Thorsson, P.; Lundström, J.N. Reduction of physiological stress by urban green space in a multisensory virtual experiment. Sci. Rep. 2019, 9, 10113. [CrossRef]

11. Gladwell, V.F.; Brown, D.K.; Barton, J.L.; Tarvainen, M.P.; Kuoppa, P.; Pretty, J.; Sandercock, G.R.H. The effects of views of nature on autonomic control. Eur. J. Appl. Physiol. 2012, 112, 3379-3386. [CrossRef]

12. Calogiuri, G.; Litleskare, S.; Fagerheim, K.A.; Rydgren, T.L.; Brambilla, E.; Thurston, M. Experiencing nature through immersive virtual environments: Environmental perceptions, physical engagement, and affective responses during a simulated nature walk. Front. Psychol. 2018, 8, 2321. [CrossRef] [PubMed]

13. De Kort, Y.A.; Meijnders, A.L.; Sponselee, A.A.; IJsselsteijn, W.A. What's wrong with virtual trees? Restoring from stress in a mediated environment. J. Environ. Psychol. 2006, 26, 309-320. [CrossRef]

14. Villani, D.; Riva, F.; Riva, G. New technologies for relaxation: The role of presence. Int. J. Stress Manag. 2007, 14, 260. [CrossRef]

15. Valtchanov, D.; Barton, K.R.; Ellard, C. Restorative effects of virtual nature settings. Cyberpsychol. Behav. Soc. Netw. 2010, 13, 503-512. [CrossRef] [PubMed]

16. Annerstedt, M.; Jönsson, P.; Wallergård, M.; Johansson, G.; Karlson, B.; Grahn, P.; Währborg, P. Inducing physiological stress recovery with sounds of nature in a virtual reality forest-Results from a pilot study. Physiol. Behav. 2013, 118, 240-250. [CrossRef]

17. Anderson, A.P.; Mayer, M.D.; Fellows, A.M.; Cowan, D.R.; Hegel, M.T.; Buckey, J.C. Relaxation with immersive natural scenes presented using virtual reality. Aerosp. Med. Hum. Perform. 2017, 88, 520-526. [CrossRef] [PubMed]

18. Browning, M.H.; Rigolon, A. School green space and its impact on academic performance: A systematic literature review. Int. J. Environ. Res. Public Health 2019, 16, 429. [CrossRef] [PubMed]

19. Trifoni, A.; Shahini, M. How does exam anxiety affect the performance of university students? Mediterr. J. Soc. Sci. 2011, 2, 93-100.

20. Putwain, D.W.; Daly, A.L.; Chamberlain, S.; Sadreddini, S. 'Sink or swim': Buoyancy and coping in the cognitive test anxiety-academic performance relationship. Educ. Psychol. 2016, 36, 1807-1825. [CrossRef]

21. Baker, J.; Ainsworth, H.; Torgerson, C.; Torgerson, D. A systematic review and meta-analysis of randomised controlled trials evaluating the effect of hypnosis on exam anxiety. Eff. Educ. 2009, 1, 27-41. [CrossRef]

22. Zeidner, M. Test Anxiety: The State of the Art; Springer Science \& Business Media: Berlin/Heidelberg, Germany, 1998.

23. Hong, E.; Karstensson, L. Antecedents of state test anxiety. Contemp. Educ. Psychol. 2002, 27, 348-367. [CrossRef] 
24. Onyeizugbo, E.U. Self-efficacy and test anxiety as correlates of academic performance. Educ. Res. 2010, 1, 477-480.

25. Abulghasemi, A. Text Anxiety, Cause, Evaluation and Treatment. Methodol. Res. 2008, 5, 82-97.

26. Duraku, Z.H. Factors influencing test anxiety among university students. Eur. J. Soc. Behav. Sci. 2017, 18, 2325. [CrossRef]

27. Parnabas, V.A.; Mahamood, Y.; Parnabas, J.; Abdullah, N.M. The relationship between relaxation techniques and sport performance. Univers. J. Psychol. 2014, 2, 108-112.

28. Nguyen, J.; Brymer, E. Nature-based guided imagery as an intervention for state anxiety. Front. Psychol. 2018, 9, 1858. [CrossRef]

29. Bratman, G.N.; Hamilton, J.P.; Hahn, K.S.; Daily, G.C.; Gross, J.J. Nature experience reduces rumination and subgenual prefrontal cortex activation. Proc. Natl. Acad. Sci. USA 2015, 112, 8567-8572. [CrossRef] [PubMed]

30. Beilock, S.L.; DeCaro, M.S. From poor performance to success under stress: Working memory, strategy selection, and mathematical problem solving under pressure. J. Exp. Psychol. Learn. Mem. Cogn. 2007, 33, 983. [CrossRef] [PubMed]

31. Krawietz, S.A. Alleviating Choking under Pressure Using Imagery; ProQuest Dissertations Publishing: Ann Arbor, MI, USA, 2013; pp. 5-13.

32. Leung, W.T.V.; Tam, T.Y.T.; Pan, W.C.; Wu, C.D.; Lung, S.C.C.; Spengler, J.D. How is environmental greenness related to students' academic performance in English and mathematics? Landsc. Urban Plan. 2019, 181, 118-124. [CrossRef]

33. Matsuoka, R.H. Student performance and high school landscapes: Examining the links. Landsc. Urban Plan. 2010, 97, 273-282. [CrossRef]

34. Kweon, B.S.; Ellis, C.D.; Lee, J.; Jacobs, K. The link between school environments and student academic performance. Urban For. Urban Green. 2017, 23, 35-43. [CrossRef]

35. Chin, E.C.; Williams, M.W.; Taylor, J.E.; Harvey, S.T. The influence of negative affect on test anxiety and academic performance: An examination of the tripartite model of emotions. Learn. Individ. Differ. 2017, 54, 1-8. [CrossRef]

36. Richardson, M.; Hunt, A.; Hinds, J.; Bragg, R.; Fido, D.; Petronzi, D.; White, M. A measure of nature connectedness for children and adults: Validation, performance, and insights. Sustainability 2019, 11, 3250. [CrossRef]

37. Nist, P.; Diehl, M. PHCC Test Anxiety Questionnaire. Available online: https://docplayer.net/24557834-Phcctest-anxiety-questionnaire.html (accessed on 21 October 2020).

38. Watson, D.; Clark, L.A.; Tellegen, A. Development and validation of brief measures of positive and negative affect: The PANAS scales. J. Personal. Soc. Psychol. 1988, 54, 1063. [CrossRef]

39. Ogundokun, M.O. Learning style, school environment and test anxiety as correlates of learning outcomes among secondary school students. IFE Psychol. Int. J. 2011, 19, 321-336. [CrossRef]

40. McAllister, S.J.; Vincent, A.; Hassett, A.L.; Whipple, M.O.; Oh, T.H.; Benzo, R.P.; Toussaint, L.L. Psychological resilience, affective mechanisms and symptom burden in a tertiary-care sample of patients with fibromyalgia. Stress Health 2015, 31, 299-305. [CrossRef]

41. Crawford, J.R.; Henry, J.D. The Positive and Negative Affect Schedule (PANAS): Construct validity, measurement properties and normative data in a large non-clinical sample. Br. J. Clin. Psychol. 2004, 43, 245-265. [CrossRef]

42. Eleven Plus Exams. (n.d.). Available online: https://www.Elevenplusexams.co.uk/assets/192/CGP11+ NonVerbalReasoningTest.pdf (accessed on 1 October 2020).

43. Gianaros, P.J.; Muth, E.R.; Mordkoff, J.T.; Levine, M.E.; Stern, R.M. A questionnaire for the assessment of the multiple dimensions of motion sickness. Aviat. Space Environ. Med. 2001, 72, 115.

44. Tarrant, J.; Viczko, J.; Cope, H. Virtual reality for anxiety reduction demonstrated by Quantitative EEG: A pilot study. Front. Psychol. 2018, 9, 1280. [CrossRef]

45. Tabachnick, B.G.; Fidell, L.S.; Ullman, J.B. Using Multivariate Statistics; Pearson: Boston, MA, USA, 2007; Volume 5.

46. Cohen, J. The Effect Size Index: D. In Statistical Power Analysis for the Behavioral Sciences; Academic Press: Cambridge, MA, USA, 1988; Volume 2.

47. Taylor, C.T.; Lyubomirsky, S.; Stein, M.B. Upregulating the positive affect system in anxiety and depression: Outcomes of a positive activity intervention. Depress. Anxiety 2017, 34, 267-280. [CrossRef] 
48. Kring, A.M.; Persons, J.B.; Thomas, C. Changes in affect during treatment for depression and anxiety. Behav. Res. Ther. 2007, 45, 1753-1764. [CrossRef] [PubMed]

49. Brown, T.A.; Chorpita, B.F.; Barlow, D.H. Structural relationships among dimensions of the DSM-IV anxiety and mood disorders and dimensions of negative affect, positive affect, and autonomic arousal. J. Abnorm. Psychol. 1998, 107, 179. [CrossRef] [PubMed]

Publisher's Note: MDPI stays neutral with regard to jurisdictional claims in published maps and institutional affiliations.

(C) 2020 by the authors. Licensee MDPI, Basel, Switzerland. This article is an open access article distributed under the terms and conditions of the Creative Commons Attribution (CC BY) license (http://creativecommons.org/licenses/by/4.0/). 